\title{
Maximal Families of Calabi-Yau Manifolds with Minimal Length Yukawa Coupling
}

\author{
Mao Sheng • Jinxing Xu • Kang Zuo
}

Received: 26 February 2013 / Accepted: 5 March 2013 / Published online: 21 March 2013

(C) School of Mathematical Sciences, University of Science and Technology of China and Springer-Verlag Berlin Heidelberg 2013

\begin{abstract}
For each natural odd number $n \geq 3$, we exhibit a maximal family of $n$-dimensional Calabi-Yau manifolds whose Yukawa coupling length is 1 . As a consequence, Shafarevich's conjecture holds true for these families. Moreover, it follows from Deligne and Mostow (Publ. Math. IHÉS, 63:5-89, 1986) and Mostow (Publ. Math. IHÉS, 63:91-106, 1986; J. Am. Math. Soc., 1(3):555-586, 1988) that, for $n=3$, it can be partially compactified to a Shimura family of ball type, and for $n=5,9$, there is a sub $\mathbb{Q}$-PVHS of the family uniformizing a Zariski open subset of an arithmetic ball quotient.
\end{abstract}

Keywords Calabi-Yau · Yukawa Coupling $\cdot$ Hodge Theory

Mathematics Subject Classification (2010) 14D07 · 14J32

\section{Introduction}

The local Torelli theorem for Calabi-Yau (abbreviated as CY) manifolds says that the Kodaira-Spencer map for a versal local deformation of a CY manifold is an isomorphism. This important fact has a consequence on the Yukawa coupling length

M. Sheng $(\bowtie) \cdot$ J. Xu

School of Mathematical Sciences, University of Science and Technology of China, Hefei 230026, China

e-mail: msheng@ustc.edu.cn

J. Xu

e-mail: xujx02@ustc.edu.cn

K. Zuo

Institut für Mathematik, Universität Mainz, Mainz 55099, Germany

e-mail:zuok@uni-mainz.de 
which is introduced in the work [13]. For a family $f: \mathcal{X} \rightarrow S$ of CY manifolds of dimension $n$, let

$$
\left(E=\bigoplus_{p+q=n} E^{p, q}, \theta=\bigoplus_{p+q=n} \theta^{p, q}\right)
$$

be the associated Higgs bundle, where $E^{p, q}=R^{q} f_{*} \Omega_{\mathcal{X} / S}^{p}$ and the Higgs field

$$
\theta^{p, q}: E^{p, q} \rightarrow E^{p-1, q+1} \otimes \Omega_{S}
$$

is given by the cup product with the Kodaira-Spencer map. The length of the Yukawa coupling $\zeta(f)$ of $f$ is then defined by

$$
\varsigma(f)=\min \left\{i \geq 1, \theta^{i}=0\right\}-1,
$$

where $\theta^{i}$ is the $i$ th iterated Higgs field

$$
\theta^{i}: E^{n, 0} \stackrel{\theta^{n, 0}}{\longrightarrow} E^{n-1,1} \otimes \Omega_{S} \stackrel{\theta^{n-1,1}}{\longrightarrow} \cdots \stackrel{\theta^{n-i+1, i-1}}{\longrightarrow} E^{n-i, i} \otimes S^{i} \Omega_{S}
$$

The local Torelli theorem implies that, for a non-isotrivial family $f$ of $n$-dimensional CY manifolds, it holds true that $1 \leq \varsigma(f) \leq n$. The connection of Yukawa coupling length with Shararevich's conjecture for CY manifolds has been intensively studied (see e.g., [5, 14]). It has been shown that, for example, if the Yukawa coupling length of $f$ is maximal, i.e., $\varsigma(f)=n$, then $f$ is rigid. The maximality of Yukawa coupling length seems to be very often for moduli spaces of CY manifolds as anti-canonical classes of a toric variety (it is the case for moduli spaces of CY manifolds with a maximal degeneration point). Our motivation is then to look for many examples of moduli spaces of CY manifolds whose Yukawa coupling lengths are minimal, i.e., 1. As far as we know, higher dimensional examples are rare in the literature. What we have obtained in this paper is an infinite series of maximal families of $n$-dimensional CY manifolds with Yukawa coupling length 1 for any odd $n \geq 3$. Here a family $f$ is said to be maximal if it is locally a versal deformation of each CY closed fiber of $f$. Our main result is summarized as follows.

Theorem 1.1 Let $n \geq 3$ be an odd number and $\mathfrak{M}_{n, n+3}$ be the moduli space of $n+3$ hyperplane arrangements of $\mathbb{P}^{n}$ in general position. Let $f_{n}: \mathcal{X}_{n} \rightarrow \mathfrak{M}_{n, n+3}$ be the family of $\frac{n+3}{2}$-fold cyclic covers of $\mathbb{P}^{n}$ branched along the $n+3$ hyperplanes in general position. Then the following statements are true:

(i) The family $f_{n}$ admits a simultaneous resolution $\tilde{f}_{n}: \tilde{\mathcal{X}}_{n} \rightarrow \mathfrak{M}_{n, n+3}$ which is a maximal family of $n$-dimensional projective $C Y$ manifolds.

(ii) $\varsigma\left(\tilde{f}_{n}\right)=1$. Consequently, Shafarevich's conjecture holds for $\tilde{f}_{n}$.

(iii) The family $\tilde{f}_{3}$ admits a partial compactification to a Shimura family over an arithmetic quotient of $\mathbb{B}^{3}$.

(iv) The families $\tilde{f}_{5}, \tilde{f}_{9}$ have a sub $\mathbb{Q}$-PVHS which uniformizes a Zariski open subset of an arithmetic ball quotient. 


\section{The Cyclic Cover and Its Crepant Resolution}

The meaning of letters in the tuple $(n, m, r)$ will be fixed throughout the paper: $n$ is a natural odd number $\geq 3, m=n+3$ and $r=\frac{m}{2}$.

Remark 2.1 The technique of this section can be applied equally to a tuple $(n, m, r)$ where $n$ is a natural number, $r$ a positive factor of $m$ and $m=n+1+\frac{m}{r}$, and yields the same result as the special case.

\subsection{The Cyclic Cover of $\mathbb{P}^{n}$}

An hyperplane arrangement $\mathfrak{A}=\left(H_{1}, \ldots, H_{m}\right)$ in $\mathbb{P}^{n}$ is said to be in general position if no $n+1$ hyperplanes in $\mathfrak{A}$ do meet. One constructs the $r$-fold cyclic cover $\pi: X \rightarrow \mathbb{P}^{n}$ branched along $H=H_{1}+\cdots+H_{m}$ as follows. For the line bundle $L=\mathcal{O}_{\mathbb{P}^{n}}(2)$ over $\mathbb{P}^{n}$, one denotes $\operatorname{Tot}(L)$ for the total space of $L$. There is the tautological section $s \in \Gamma\left(\operatorname{Tot}(L), p^{*} L\right)$ of the pull-back of $L$ via the natural projection $p: \operatorname{Tot}(L) \rightarrow \mathbb{P}^{n}$. Suppose the hyperplane $H_{i}, 1 \leq i \leq m$ is defined as the zero locus of the section $s_{i} \in \Gamma\left(\mathbb{P}^{n}, \mathcal{O}(1)\right)$, then we have the pull-back section $p^{*} s_{i} \in \Gamma\left(\operatorname{Tot}(L), p^{*} \mathcal{O}(1)\right)$. The $r$-fold cover $X$ is defined as the zero locus of the section

$$
s^{r}-p^{*} s_{1} \otimes \cdots \otimes p^{*} s_{m} \in \Gamma\left(\operatorname{Tot}(L), p^{*} \mathcal{O}(m)\right) .
$$

The natural projection $p$ induces the one $\pi: X \rightarrow \mathbb{P}^{n}$. It is generically étale and Galois, whose Galois group is cyclic of order $r$, and singular over $H$. The variety $X$ is projective with trivial $K_{X}$. It is a singular CY variety whose singular locus under $\pi$ is exactly the singularity of $H$.

\subsection{The Crepant Resolution}

In this paragraph we aim to obtain a good smooth model of the cyclic cover $X$. First recall the order function on a smooth variety. Let $M$ be a smooth variety over an algebraically closed field and $\mathcal{I}$ be an ideal sheaf on $M$. For any point $x \in M$, the order of $\mathcal{I}$ at $x$ is defined as $\operatorname{Ord}_{x} \mathcal{I}:=\max \left\{r \mid \mathcal{I}_{x} \subset m_{x}^{r} \mathcal{O}_{M, x}\right\}$, where $m_{x}$ is the maximal ideal of the local ring $\mathcal{O}_{M, x}$. For a smooth and irreducible closed subvariety $Z$ of $M$, the order of $\mathcal{I}$ along $Z$ is defined as $\operatorname{Ord}_{Z} \mathcal{I}:=\operatorname{Ord}_{p} \mathcal{I}$, where $p \in Z$ is the generic point of $Z$. We have the following well-known formula for the canonical bundle under a blow-up.

Lemma 2.2 Let $M$ be a smooth variety over an algebraically closed field and $X \subset M$ be a (possibly singular and non-reduced) hypersurface. Suppose $\mathcal{I}(X)$ is the defining ideal sheaf of $X$ in $M$. Let $Z \subset X$ be a smooth and irreducible closed subvariety of $M$, with codimension $\operatorname{codim}(Z, M)=n$. Suppose that the order of $\mathcal{I}(X)$ along $Z$ is $\operatorname{Ord}_{Z} \mathcal{I}(X)=r$. Consider the blow-up of $M$ along $Z: \tilde{M} \stackrel{\tilde{\pi}}{\rightarrow} M$. Let $\tilde{X}=B l_{Z} X \stackrel{\pi}{\rightarrow} X$ be the strict transform of $X$ and $E=\tilde{\pi}^{-1}(Z)$ the exceptional divisor. Then we have the following formula relating the canonical bundles of $\tilde{X}$ and $X$ :

$$
K_{\tilde{X}} \simeq \pi^{*} K_{X}+\left.(n-r-1) \mathcal{O}_{\tilde{M}}(E)\right|_{\tilde{X}}
$$


In particular, if $\operatorname{codim}(Z, M)=2$ and $\operatorname{Ord}_{Z} \mathcal{I}(X)=1$, then $K_{\tilde{X}} \simeq \pi^{*} K_{X}$.

Proof The proof is a direct application of the adjunction formula.

In order to fix notation, we recall the following definition.

Definition 2.3 If $M$ is a smooth variety, and $E_{1}, \ldots, E_{k}$ are divisors of $M$, we say $E_{1}, \ldots, E_{k}$ meet transversally at a closed point $x \in M$ if one can choose a regular system of parameters $z_{1}, \ldots, z_{l} \in \mathcal{O}_{M, x}$ at $x$ such that for each $1 \leq i \leq k$

(1) either $x \notin E_{i}$, or

(2) $E_{i}=\left(z_{c(i)}=0\right)$ in a neighborhood of $x$ for some $c(i)$, and

(3) $c(i) \neq c\left(i^{\prime}\right)$ if $i \neq i^{\prime}$.

and the regular system of parameters $z_{1}, \ldots, z_{l} \in \mathcal{O}_{M, x}$ is called a coordinate system at $x$ admissible to $E_{1}, \ldots, E_{k} . E_{1}, \ldots, E_{k}$ are said to meet transversally if they meet transversally at each closed point of $M$. In this case, $D=\sum_{i=1}^{k} E_{i}$ is called a simple normal crossing divisor on $M$ and a subvariety $Z \subset M$ is said to meet transversally with $E_{1}, \ldots, E_{k}$ if at each closed point $x \in Z$, one can choose $z_{1}, \ldots, z_{l}$ as above such that in addition

(4) $Z=\left(z_{j_{1}}=\cdots=z_{j_{s}}=0\right)$ for some $j_{1}, \ldots, j_{s}$, again in some open neighborhood of $x$.

In particular, $Z$ is smooth, and some of the $E_{i}$ are allowed to contain $Z$.

Let $M$ be a smooth variety, and $\mathfrak{E}=\left\{E_{1}, \ldots, E_{s}\right\}, \mathfrak{F}=\left\{F_{1}, \ldots, F_{t}\right\}$ be sets of smooth divisors of $M$ such that the $s+t$ divisors $E_{1}, \ldots, E_{s}, F_{1}, \ldots, F_{t}$ meet transversally on $M$. A reduced and irreducible hypersurface $X \subset M$ is called pre-binomial with respect to $(\mathfrak{E}, \mathfrak{F})$ if for any closed point $x \in X$,

(1) either $X, E_{1}, \ldots, E_{s}, F_{1}, \ldots, F_{t}$ meet transversally at $x$, or

(2) there exists a coordinate system $\left(y_{1}, \ldots, y_{m}, x_{1}, \ldots, x_{n}\right)$ at $x$ admissible to $E_{1}, \ldots, E_{s}, F_{1}, \ldots, F_{t}$ (refer to Definition 2.3 for the notation) such that

- the defining equation of $X$ is

$$
y_{1}^{a_{1}} \cdots y_{p}^{a_{p}}-x_{1} \cdots x_{q}=0
$$

in a nonempty open neighborhood of $x$, where the integers satisfy

$$
1 \leq p \leq m, \quad 1 \leq q \leq n, \quad a_{1} \geq 1, \quad \ldots, \quad a_{p} \geq 1,
$$

and

- for each $1 \leq i \leq p, y_{i}=0$ is a defining equation for some $E_{c_{i}} \in \mathfrak{E}$ in a nonempty open neighborhood of $x$, and

- for each $1 \leq j \leq q, x_{j}=0$ is a defining equation for some $F_{d_{j}} \in \mathfrak{F}$ in a nonempty open neighborhood of $x$. 
If $X$ is a pre-binomial hypersurface of $M$ with respect to $(\mathfrak{E}, \mathfrak{F})$, keeping the notations in the above definition, then for any closed point $x \in X$, for any $E \in \mathfrak{E}, F \in \mathfrak{F}$, define

$$
\begin{aligned}
& e(E, x)= \begin{cases}a_{i}, & \text { if } E=E_{c_{i}} \text { for some } i \text { in the above definition; } \\
0, & \text { otherwise, }\end{cases} \\
& e(F, x)= \begin{cases}1, & \text { if } F=F_{d_{j}} \text { for some } j \text { in the above definition; } \\
0, & \text { otherwise. }\end{cases}
\end{aligned}
$$

It is not difficult to verify that the above definitions of $e(E, x), e(F, x)$ do not depend on the choice of local coordinates, so they are well-defined nonnegative integers.

Let $M, \mathfrak{E}, \mathfrak{F}$ be as above, then a hypersurface $X$ of $M$ is called binomial with respect to $(\mathfrak{E}, \mathfrak{F})$ if it is pre-binomial with respect to $(\mathfrak{E}, \mathfrak{F})$ and satisfies the following two additional conditions:

(3) $\forall E \in \mathfrak{E} \cup \mathfrak{F}, \forall$ closed points $x_{1}, x_{2} \in E \cap X, e\left(E, x_{1}\right)=e\left(E, x_{2}\right)$.

(4) $\forall E \in \mathfrak{E}, \forall F \in \mathfrak{F}$, if there exists a closed point $x \in X$ such that $e(E, x)>0$ and $e(F, x)>0$, then $E \cap F \subset X$.

Our key observation is the following stable proposition for binomial hypersurfaces.

Proposition 2.4 Let $M, \mathfrak{E}=\left\{E_{1}, \ldots, E_{s}\right\}, \mathfrak{F}=\left\{F_{1}, \ldots, F_{t}\right\}$ be as above. Suppose $X$ is a hypersurface of $M$ binomial with respect to $(\mathfrak{E}, \mathfrak{F})$ and there exists a closed point $x \in X$ such that $e\left(E_{1}, x\right)>0$ and $e\left(F_{1}, x\right)>0$. Let $M_{1}=B l_{Z} M \stackrel{\pi}{\rightarrow} M$ be the blow-up of $M$ along $Z=E_{1} \cap F_{1} \subset X$ and $X_{1}, E_{1}^{\prime}, \ldots, E_{s}^{\prime}, F_{1}^{\prime}, \ldots, F_{t}^{\prime}$ be the strict transforms of $X, E_{1}, \ldots, E_{s}, F_{1}, \ldots, F_{t}$, respectively. Let $\mathfrak{E}_{1}=\left\{E_{1}^{\prime}, \ldots, E_{s}^{\prime}, E_{s+1}^{\prime}\right\}$, $\mathfrak{F}_{1}=\left\{F_{1}^{\prime}, \ldots, F_{t}^{\prime}\right\}$, where $E_{s+1}^{\prime}=\pi^{-1}(Z)$ is the exceptional divisor. Then

(1) $\mathfrak{E}_{1} \cup \mathfrak{F}_{1}$ is a set of smooth divisors meeting transversally on $M_{1}$, and $X_{1}$ is a hypersurface of $M_{1}$ binomial with respect to $\left(\mathfrak{E}_{1}, \mathfrak{F}_{1}\right)$.

(2) Each irreducible component of the singular locus of $X$, say $\operatorname{Sing}(X)$, has the form $E_{i_{1}} \cap F_{j_{1}} \cap F_{j_{2}} \subset X$ or $E_{i_{1}} \cap E_{i_{2}} \cap F_{j_{1}} \cap F_{j_{2}} \subset X$, for $1 \leq i_{1} \neq i_{2} \leq s$, $1 \leq j_{1} \neq j_{2} \leq t$.

(3) For the induced morphism $X_{1} \stackrel{\pi_{1}}{\rightarrow} X$ we have $\pi_{1}^{-1}\left(E_{1} \cap F_{1} \cap \operatorname{Sing}(X)\right) \rightarrow E_{1} \cap$ $F_{1} \cap \operatorname{Sing}(X)$ is a $\mathbb{P}^{1}$-bundle, and $\pi_{1}^{-1}\left(X-E_{1} \cap F_{1} \cap \operatorname{Sing}(X)\right) \rightarrow X-E_{1} \cap$ $F_{1} \cap \operatorname{Sing}(X)$ is an isomorphism.

(4) Let $\mathfrak{T}=\left\{E_{i_{1}}^{\prime}, \ldots, E_{i_{k}}^{\prime}, F_{j_{1}}^{\prime}, \ldots, F_{j_{l}}^{\prime}\right\} \subset \mathfrak{E}_{1} \cup \mathfrak{F}_{1}$ be a subset of $\mathfrak{E}_{1} \cup \mathfrak{F}_{1}$ satisfying $V=E_{i_{1}}^{\prime} \cap \cdots \cap E_{i_{k}}^{\prime} \cap F_{j_{1}}^{\prime} \cap \cdots \cap F_{j_{l}}^{\prime} \subset X_{1}$, then $V=\emptyset$ if $\left\{E_{1}^{\prime}, F_{1}^{\prime}\right\} \subset \mathfrak{T}$. Suppose $V \neq \emptyset$, then

$$
\pi(V)= \begin{cases}E_{i_{1}} \cap \cdots \cap E_{i_{k}} \cap F_{j_{1}} \cap \cdots \cap F_{j_{l}}, & \text { if } E_{s+1}^{\prime} \notin \mathfrak{T} ; \\ E_{1} \cap F_{1} \cap E_{i_{2}} \cap \cdots \cap E_{i_{k}} \cap F_{j_{1}} \cap \cdots \cap F_{j_{l}}, & \text { if } E_{i_{1}}^{\prime}=E_{s+1}^{\prime} .\end{cases}
$$

(5) Notations as in (4). If $\left\{E_{1}^{\prime}, F_{1}^{\prime}\right\} \cap \mathfrak{T} \neq \emptyset$, then the induced morphism $V \stackrel{\tilde{\pi}}{\rightarrow} \pi(V)$ is an isomorphism. If $\left\{E_{1}^{\prime}, F_{1}^{\prime}\right\} \cap \mathfrak{T}=\emptyset$, then the induced morphism $V \stackrel{\tilde{\pi}}{\rightarrow} \pi(V)$ satisfies that $\tilde{\pi}^{-1}\left(E_{1} \cap F_{1} \cap \pi(V)\right) \rightarrow E_{1} \cap F_{1} \cap \pi(V)$ is a $\mathbb{P}^{1}$-bundle, and $\tilde{\pi}^{-1}\left(\pi(V)-E_{1} \cap F_{1}\right) \rightarrow \pi(V)-E_{1} \cap F_{1}$ is an isomorphism. 
Proof The verification is straightforward in local coordinates.

Let $M, \mathfrak{E}=\left\{E_{1}, \ldots, E_{s}\right\}, \mathfrak{F}=\left\{F_{1}, \ldots, F_{t}\right\}$ be as above. Suppose $X$ is a hypersurface of $M$ binomial with respect to $(\mathfrak{E}, \mathfrak{F})$. In order to get a resolution of $X$, we define a function on $X$ to measure the singularities on it. For any closed point $x \in X$, define $g_{1}(x)=\sum_{F \in \mathfrak{F}} e(F, x), g_{2}(x)=\sum_{E \in \mathfrak{E}} e(E, x)$. Then we get a function on $X$ :

$$
g: X \rightarrow \mathbb{N} \times \mathbb{N}, \quad x \mapsto\left(g_{1}(x), g_{2}(x)\right) .
$$

It is easy to see that if $g(x) \neq(0,0)$, then $g^{-1}(g(x))$ is a disjoint union of smooth subvarieties of $X$. In this case, let $n(x)$ be the number of irreducible components of $g^{-1}(g(x))$. Then we define the following function:

$$
\begin{aligned}
& f: X \rightarrow \mathbb{N} \times \mathbb{N} \times \mathbb{N} \\
& f(x)= \begin{cases}\left(g_{1}(x), g_{2}(x), n(x)\right), & \text { if } g(x) \neq(0,0) ; \\
0, & \text { if } g(x)=(0,0) .\end{cases}
\end{aligned}
$$

Given the lexicographic order, $\mathbb{N} \times \mathbb{N} \times \mathbb{N}$ is a well-ordered set, i.e., for

$$
\left(a_{1}, b_{1}, c_{1}\right), \quad\left(a_{2}, b_{2}, c_{2}\right) \in \mathbb{N} \times \mathbb{N} \times \mathbb{N},
$$

$\left(a_{1}, b_{1}, c_{1}\right)<\left(a_{2}, b_{2}, c_{2}\right)$ if and only if $a_{1}<a_{2}$, or $a_{1}=a_{2}$ and $b_{1}<b_{2}$, or $a_{1}=a_{2}$ and $b_{1}=b_{2}$ and $c_{1}<c_{2}$. Then it is easy to see that $f$ is an upper semi-continuous function on $X$. Therefore we get the following algorithm $(*)$ to resolve the singularity of $X$ :

(0) If the maximal value $\max _{x \in X} f(x)=(0,0,0)$, then $X$ is already a smooth variety meeting transversally with the divisors in $\mathfrak{E} \cup \mathfrak{F}$.

(1) If $\max _{x \in X} f(x)>(0,0,0)$, take any closed point $x \in X$ such that $f(x)$ attains the maximal value of $f$. It is not difficult to see that we can choose $E_{i} \in \mathfrak{E}, F_{j} \in \mathfrak{F}$ such that $e\left(E_{i}, x\right)>0$ and $e\left(F_{j}, x\right)>0$. Then blow up $M$ along $Z=E_{i} \cap F_{j}$ (we have $Z=E_{i} \cap F_{j} \subset X$ by the definition of binomial hypersurfaces). Let $E$ be the exceptional divisor. Let $M_{1}=B l_{Z} M$, and $X_{1}, E_{1}^{\prime}, \ldots, E_{s}^{\prime}, F_{1}^{\prime}, \ldots, F_{t}^{\prime}$ be the strict transforms of $X, E_{1}, \ldots, E_{s}, F_{1}, \ldots, F_{t}$, respectively. Let $\mathfrak{E}_{1}=\left\{E, E_{1}^{\prime}, \ldots, E_{s}^{\prime}\right\}, \mathfrak{F}_{1}=\left\{F_{1}^{\prime}, \ldots, F_{t}^{\prime}\right\}$, then according to Proposition 2.4, $X_{1}$ is a hypersurface of $M_{1}$ binomial with respect to $\left(\mathfrak{E}_{1}, \mathfrak{F}_{1}\right)$. So we can define a function $f_{1}: X_{1} \rightarrow \mathbb{N} \times \mathbb{N} \times \mathbb{N}$ in the same way as above. Let $\pi_{1}: X_{1} \rightarrow X$ be the blow-up morphism. Then it is direct to verify:

- for any point $x \in X_{1}, f_{1}(x) \leq f\left(\pi_{1}(x)\right)$, and

- the maximal value drops strictly, i.e. $\max _{x \in X_{1}} f_{1}(x)<\max _{x \in X} f(x)$.

Note also that since $Z$ has codimension 2 everywhere in $M$, and $X$ has order 1 at the generic point of each irreducible component of $Z$, we have $K_{X_{1}} \simeq \pi_{1}^{*} K_{X}$, according to Lemma 2.2.

(2) If $\max _{x \in X_{1}} f_{1}(x)>(0,0,0)$, then continue to blow up $M_{1}$ and get

$$
M_{2}, \quad X_{2}, \quad \mathfrak{E}_{2}, \quad \mathfrak{F}_{2}, \quad f_{2} .
$$


We summarize the above discussions in the following theorem.

Theorem 2.5 Let $M, \mathfrak{E}=\left\{E_{1}, \ldots, E_{s}\right\}, \mathfrak{F}=\left\{F_{1}, \ldots, F_{t}\right\}$ be as above. Suppose $X$ is a hypersurface of $M$ binomial with respect to $(\mathfrak{E}, \mathfrak{F})$. Then the above algorithm (*) terminates after finite steps. Suppose it terminates after $N$ steps, then we get $M_{N}, X_{N}, \mathfrak{E}_{N}, \mathfrak{F}_{N}$ such that $X_{N}$ is a smooth hypersurface of $M_{N}$ meeting transversally with the set of divisors $\mathfrak{E}_{N} \cup \mathfrak{F}_{N}$. Moreover, let $\pi=\pi_{N} \circ \cdots \circ \pi_{1}: X_{N} \rightarrow X$ be the composition of blow-up morphisms, then

- $\pi$ is crepant, i.e. $K_{X_{N}} \simeq \pi^{*} K_{X}$;

- $\pi$ is a strong resolution of $X$, i.e. if $U=X-\operatorname{Sing}(X)$ is the regular part of $X$, then $\pi$ induces an isomorphism $\pi^{-1}(U) \stackrel{\sim}{\rightarrow} U$;

- $\pi$ is a projective morphism, moreover, it is a composition of blow-ups along smooth centers.

Proof Most of the theorem follows from the above discussions. We just explain why $\pi$ is a strong resolution. Note that in each blow-up step the smooth center $Z_{i}$ is contained completely in $X_{i}$. So in the regular part of $X_{i}$, we just blow up a Cartier divisor. Therefore, the regular part of $X_{i}$ remains unchanged.

Now we give an application of Theorem 2.5. Suppose $Q$ is a smooth variety, and $D=\sum_{j=1}^{t} F_{j}$ is a simple normal crossing divisor on $Q$ defined by the section $s_{D} \in \Gamma\left(Q, \mathcal{O}_{Q}(D)\right)$. Let $a$ be a positive integer and $L \in \operatorname{Pic}(Q)$ such that $a L=D$. Then in the total space of $L: M=\operatorname{Tot}(L) \stackrel{p}{\rightarrow} Q$, we have the tautological section $s_{0} \in$ $\Gamma\left(M, p^{*} L\right)$. The hypersurface $X$ of $M$ defined by the equation $s_{0}^{a}=p^{*} s_{D}$ is called the $a$-fold cyclic cover of $Q$ branched along $D$. It is easy to verify that $X$ is a hypersurface of $M$ binomial with respect to $(\mathfrak{E}, \mathfrak{F})=\left(\left\{E_{0}=\left(s_{0}=0\right)\right\},\left\{p^{*} F_{1}, \ldots, p^{*} F_{t}\right\}\right)$. So we can apply Theorem 2.5 to get a crepant resolution of $X$. That is, we have the following corollary.

Corollary 2.6 Suppose $Q$ is a smooth variety and $D=\sum_{j=1}^{t} F_{j}$ is a simple normal crossing divisor on $Q$, for any $a \geq 1$, if there exists $L \in \operatorname{Pic}(Q)$ such that $a L=D$, then the a-fold cyclic cover of $Q$ branched along $D$ admits a crepant resolution, which can be obtained by applying the crepant resolution algorithm $(*)$.

For the cyclic cover $X$ constructed in Sect. 2.1, we can simply apply the above result to obtain a crepant resolution $\sigma: \tilde{X} \rightarrow X$.

\subsection{The Middle Cohomology Does Not Change Under Resolution}

In this paragraph we show that $\sigma: \tilde{X} \rightarrow X$ induces an isomorphism on the middle cohomologies. To start with, we show first that the Hodge structure $H^{n}(X, \mathbb{Q})$ is actually pure. This is mostly easy if one notices that there is another CY, $Y$ arising from the same hyperplane arrangement as $X$, which is indeed smooth. We follow [3, Sect. 2.2] for the construction of $Y$, the Kummer cover associated with $\mathfrak{A}$. Let 
$A$ be a matrix whose columns define the hyperplane arrangement $\mathfrak{A}$ and $B=\left(b_{i j}\right)$ a matrix fitting into a short exact sequence

$$
0 \rightarrow \mathbb{C}^{m-2} \stackrel{A}{\longrightarrow} \mathbb{C}^{m} \stackrel{B}{\longrightarrow} \mathbb{C}^{2} \rightarrow 0
$$

We define $Y$ to be the complete intersection of $\frac{m}{r}=2$ degree $r$ hypersurfaces in $\mathbb{P}^{m-1}=\operatorname{Proj} \mathbb{C}\left[z_{0}: \cdots: z_{m-1}\right]$ defined by

$$
b_{i 1} z_{0}^{r}+\cdots+b_{i m} z_{m-1}^{r}=0, \quad i=1,2 .
$$

Using the Jacobian criterion, one sees easily that $Y$ is smooth, and by the adjunction formula has trivial canonical bundle. The structure of $Y$ as a Kummer cover is seen as follows. Put $G_{1}=\bigoplus_{i=1}^{m} \mathbb{Z} / r / \triangle(\mathbb{Z} / r)$. Here $\triangle$ denotes for the diagonal embedding $\mathbb{Z} / r \rightarrow \bigoplus_{i=1}^{m} \mathbb{Z} / r$. For $a=\left(a_{0}, \ldots, a_{m}\right) \in G_{1}$, we define an automorphism $\sigma_{a}: \mathbb{P}^{m-1} \rightarrow \mathbb{P}^{m-1}$ by

$$
\sigma_{a}\left(z_{0}: \cdots: z_{m-1}\right)=\left(\zeta_{r}^{a_{0}} z_{0}: \cdots: \zeta_{r}^{a_{m-1}} z_{m-1}\right),
$$

where $\zeta_{r}$ is a primitive $r$ th root of unit. The matrix $A$ defines a linear embedding $j: \mathbb{P}^{n} \rightarrow \mathbb{P}^{m-1}$ of projective spaces. Similar to Lemma 2.4 loc. cit., one sees that the map

$$
\mathbb{P}^{m-1} \rightarrow \mathbb{P}^{m-1}, \quad\left(z_{0}: \cdots: z_{m-1}\right) \mapsto\left(z_{0}^{r}: \cdots: z_{m-1}^{r}\right)
$$

realizing $Y$ as the Kummer cover $Y \rightarrow j\left(\mathbb{P}^{n}\right)$ with Galois group $G_{1}$ and with branch locus $j(H)$. The group $G_{1}$ contains a distinguished normal subgroup $N_{1} \triangleleft G_{1}$ of index $r$, the kernel of the map $a \mapsto \sum_{i=0}^{m-1} a_{i}$. We state the following result whose proof is referred to Proposition 2.5 loc. cit.

Lemma 2.7 We have $X \simeq Y / N_{1}$. Consequently, there is an isomorphism of pure polarized $\mathbb{Q}$-Hodge structures

$$
H^{n}(X, \mathbb{Q}) \simeq H^{n}(Y, \mathbb{Q})^{N_{1}}
$$

Proposition 2.8 If $p, q \geq 0$ and $p \neq q$, then $\sigma$ induces an isomorphism

$$
\sigma^{*}: H^{p, q}(X) \stackrel{\sim}{\rightarrow} H^{p, q}(\tilde{X}) \text {. }
$$

In particular, one has the isomorphism of $\mathbb{Q}-P V H S$ :

$$
\sigma^{*}: H^{n}(X, \mathbb{Q}) \stackrel{\sim}{\rightarrow} H^{n}(\tilde{X}, \mathbb{Q}) .
$$

We need some lemmas.

Lemma 2.9 Let $f: \tilde{X} \rightarrow X$ be a proper modification with discriminant $D$. Put $E=$ $f^{-1}(D)$. For $p, q \geq 0$, if $H^{p, q}(X)=H^{p, q}(E)=0$, then $H^{p, q}(\tilde{X})=0$.

Proof This follows directly from [10, Corollary-Definition 5.37]. 
Lemma 2.10 Let $\pi: \tilde{V} \rightarrow V$ be a surjective morphism between projective varieties, $Z \subset V$ a closed subvariety such that $\pi^{-1}(Z) \stackrel{\pi}{\rightarrow} Z$ is a $\mathbb{P}^{1}$-bundle and $\pi^{-1}(V-$ $Z) \stackrel{\pi}{\rightarrow} V-Z$ is an isomorphism. For $p, q \geq 0$ and $p \neq q$, if $H^{p, q}(Z)=0$, then the natural homomorphism $H^{p, q}(V) \stackrel{\pi^{*}}{\rightarrow} H^{p, q}(\tilde{V})$ is surjective.

Proof This follows from Lemma 2.9 and the Leray-Hirsch Theorem for the $\mathbb{P}^{1}$-bundle $\pi^{-1}(Z) \stackrel{\pi}{\rightarrow} Z$.

We come to the proof of Proposition 2.8.

Proof Set $M=\operatorname{Tot}(L)$ and $E_{1}=\{s=0\}, F_{j}=\left\{p^{*} s_{j}=0\right\}$ divisors in $M$. Put

$$
\mathfrak{E}=\left\{E_{1}\right\}, \quad \mathfrak{F}=\left\{F_{1}, \ldots, F_{m}\right\} .
$$

Then $X$ is a binomial hypersurface of $M$ with respect to $(\mathfrak{E}, \mathfrak{F})$. The crepant resolution algorithm applied to the data $X, M,(\mathfrak{E}, \mathfrak{F})$ provides us with the blow-up sequence

$$
\tilde{X}=X_{N} \stackrel{\sigma_{N}}{\longrightarrow} X_{N-1} \stackrel{\sigma_{N-2}}{\longrightarrow} \cdots \stackrel{\sigma_{1}}{\longrightarrow} X
$$

such that for any $i=1,2, \ldots, N, X_{i}$ is a binomial hypersurface of $M_{i}$ with respect to $\left(\mathfrak{E}_{i}, \mathfrak{F}_{i}\right)$, and $\tilde{X}=X_{N}$ is smooth and meet transversally to the divisors in $\mathfrak{E}_{N} \cup \mathfrak{F}_{N}$. An induction using Proposition 2.4 and Lemma 2.10 shows that for any $i=1,2, \ldots, N$, for any elements $D_{1}, \ldots, D_{k} \in \mathfrak{E}_{i} \cup \mathfrak{F}_{i}$, if $D_{1} \cap \cdots \cap D_{k} \subset X_{i}$, then $H^{p, q}\left(D_{1} \cap\right.$ $\left.\cdots \cap D_{k}\right)=0$, for any $p, q \geq 0$ and $p \neq q$. Then a further induction using (3) of Proposition 2.4 and Lemma 2.10 shows that $H^{p, q}(X) \stackrel{\sigma^{*}}{\rightarrow} H^{p, q}(\tilde{X})$ is surjective, for any $p, q \geq 0$ and $p \neq q$, where $\sigma=\sigma_{N} \circ \cdots \circ \sigma_{1}$. [10, Theorem 5.41] shows that $H^{p, q}(X) \stackrel{\sigma^{*}}{\rightarrow} H^{p, q}(\tilde{X})$ is injective, for any $p, q \geq 0$. So we finally find that $H^{p, q}(X) \stackrel{\sigma^{*}}{\rightarrow} H^{p, q}(\tilde{X})$ is an isomorphism, for any $p, q \geq 0$ and $p \neq q$.

Corollary 2.11 Let $\pi: X \rightarrow \mathbb{P}^{n}$ be the $r$-fold cyclic cover of $\mathbb{P}^{n}$ branched along $m$ hyperplanes in general position in Sect. 2.1. Let $\tilde{X} \stackrel{\sigma}{\rightarrow} X$ be the crepant resolution constructed after Corollary 2.6. The obtained $\tilde{X}$ is a smooth projective CY manifold.

Proof By Theorem 2.5, $\tilde{X}$ is smooth. Also, as the morphism $\sigma: \tilde{X} \rightarrow X$ is projective by the same result, $\tilde{X}$ is projective whose canonical bundle is trivial. By Proposition 2.8 , for any $0<i<n$, we have

$$
H^{i}\left(\tilde{X}, \mathcal{O}_{\tilde{X}}\right) \simeq H^{0, i}(\tilde{X}) \simeq H^{0, i}(X)=H^{0, i}(Y)^{N_{1}}=0 .
$$

This shows the result.

\section{The Hodge Structure of the Cyclic Cover}

Let $\pi: X \rightarrow \mathbb{P}^{n}$ be the $r$-fold cyclic cover branched along $H=\sum_{i=1}^{m} H_{i}$ where $\left\{H_{1}, \ldots, H_{m}\right\}$ is a hyperplane arrangement of $\mathbb{P}^{n}$ in general position. In this section we investigate the Hodge structure $H^{n}(X, \mathbb{Q})$. We first record its Hodge numbers. 
Lemma 3.1 Let $h^{p, q}=\operatorname{dim} H^{p, q}(X)$ for $p, q \geq 0$ and $p+q=n$. One has

$$
h^{p, q}(X)= \begin{cases}q+1, & \text { if } q \text { is even } \\ n+1-q, & \text { if } q \text { is odd }\end{cases}
$$

Proof By Lemma 2.7, we can derive the Hodge numbers of $X$ from those of $Y$, which is a smooth complete intersection. By the work of Terasoma [12], one can represent the cohomology classes of $Y$ by a certain Jacobian ring, together with an explicit description of the action of $G_{1}$. After the computation has been implemented, we found that [2, Lemma 8.2] actually contains our result (set $\mu=\left(\frac{1}{r}, \ldots, \frac{1}{r}\right)$ in the cited lemma). The detail is therefore omitted.

\subsection{The Cyclic Cover of $\mathbb{P}^{1}$ Branched Along $m$ Distinct Points}

For $m$ distinct points $p_{i}, 1 \leq i \leq m$ in $\mathbb{P}^{1}$, we consider the $r$-fold cyclic cover of $\mathbb{P}^{1}$ branched along $\sum_{i} p_{i}$. Call it the curve $C$, and let $p: C \rightarrow \mathbb{P}^{1}$ be the natural projection. Fix a generator $\iota \in \operatorname{Aut}\left(C \mid \mathbb{P}^{1}\right)$. Let $\mathbb{Q}\left(\zeta_{r}\right) \subset \mathbb{C}$ be the $r$ th cyclotomic field. The induced action $\iota^{*}$ on $V_{\mathbb{Q}}:=H^{1}(C, \mathbb{Q})$ induces a decomposition of $V_{\mathbb{Q}\left(\zeta_{r}\right)}:=V_{\mathbb{Q}} \otimes \mathbb{Q}\left(\zeta_{r}\right)$ into direct sum of $\mathbb{Q}\left(\zeta_{r}\right)$-PHSs:

$$
V_{\mathbb{Q}\left(\zeta_{r}\right)}=\bigoplus_{i=0}^{r-1} V_{i}
$$

where $V_{i}$ is the eigenspace of $\iota^{*}$ with eigenvalue $\zeta_{r}^{i}$. The following lemma is well known.

Lemma 3.2 Let $V_{i} \otimes \mathbb{C}=V_{i}^{1,0} \oplus V_{i}^{0,1}$ be the induced Hodge decomposition from the weight 1 PHS of $C$. We have $\overline{V_{i}^{1,0}}=V_{r-i}^{0,1}$ and

$$
\operatorname{dim} V_{0}=0, \quad \operatorname{dim} V_{i}^{1,0}=2 i-1, \quad 1 \leq i \leq r-1 .
$$

Proof See for example [6, Lemma 4.2].

The next lemma is purposed for a later use.

Lemma 3.3 The weight $n \mathbb{Q}$-PHS $\wedge^{n} V_{\mathbb{Q}}$ admits a decomposition

$$
\bigwedge^{n} V_{\mathbb{Q}}=W_{1, \mathbb{Q}} \oplus W_{2, \mathbb{Q}}
$$

with $W_{1, \mathbb{Q}} \otimes \mathbb{Q}\left(\zeta_{r}\right)=\bigoplus_{i=1}^{r-1} \bigwedge^{n} V_{i}$. Furthermore, only for $n=3,5,9$, one has a further decomposition of $\mathbb{Q}$-PHS

$$
W_{1, \mathbb{Q}}=W_{\text {unif, } \mathbb{Q}} \oplus W_{1, \mathbb{Q}}^{\prime}
$$


such that

$$
W_{\text {unif }, \mathbb{Q}} \otimes \mathbb{Q}\left(\zeta_{r}\right)=\bigwedge^{n} V_{1} \oplus \bigwedge^{n} V_{r-1}
$$

Proof Consider the Galois action on the decomposition

$$
\begin{aligned}
\bigwedge^{n}\left(V_{\mathbb{Q}\left(\zeta_{r}\right)}\right) & =\bigwedge^{n}\left(\bigoplus_{i} V_{i}\right) \\
& =\bigoplus_{i} \bigwedge^{n} V_{i} \oplus \bigoplus_{I,|I| \geq 2} \bigwedge^{r_{I}} V_{I} .
\end{aligned}
$$

Here $I=\left(i_{1}, \ldots, i_{|I|}\right)$ for $1 \leq i_{1}<\cdots<i_{|I|} \leq n$ is a multi-index, $r_{I}=\left(r_{1}, \ldots, r_{|I|}\right)$ is a sequence of nonnegative integers satisfying $\sum r_{i}=n$ and

$$
\bigwedge^{r_{I}} V_{I}=\bigwedge^{r_{1}} V_{i_{1}} \otimes \cdots \otimes \bigwedge^{r_{|I|}} V_{i_{|I|}}
$$

It is clear that $\bigoplus_{i} \bigwedge^{n} V_{i}$ is invariant under the Galois action. So is the other factor. Thus they underlie $\mathbb{Q}$-subspaces of $\bigwedge^{n} V_{\mathbb{Q}}$. Consider furthermore the Galois orbit of $\bigwedge^{n} V_{1}$. It contains at least $\bigwedge^{n} V_{r-1}$, and $\bigwedge^{n} V_{1} \oplus \bigwedge^{n} V_{r-1}$ has a sub $\mathbb{Q}$-structure iff the Galois orbit contains no more direct summand. As the Galois group is isomorphic to the unit group of $\mathbb{Z} / r$ and it is an elementary fact that the unit group is of order two only if $r=3,4,6$, it follows that only when $n=3,5,9, \bigwedge^{n} V_{1} \oplus \bigwedge^{n} V_{r-1}$ underlie a sub $\mathbb{Q}$-structure. The lemma is proved.

Now we perform a similar construction to the one taken in [3, Sect. 2.3]. Let $\gamma$ : $\left(\mathbb{P}^{1}\right)^{n} \rightarrow \operatorname{Sym}^{n}\left(\mathbb{P}^{1}\right)=\mathbb{P}^{n}$ be the Galois cover with Galois group $S_{n}$, the permutation group of $n$ letters, and the identification attaches to a divisor of degree $n$ the ray of its equation in $H^{0}\left(\mathbb{P}^{1}, \mathcal{O}(n)\right)$.

Lemma 3.4 Put $H_{i}=\gamma\left(\left\{p_{i}\right\} \times\left(\mathbb{P}^{1}\right)^{n-1}\right)$. Then $\left(H_{1}, \ldots, H_{m}\right)$ is a hyperplane arrangement in $\mathbb{P}^{n}$ in general position.

Proof The divisors of degree $n$ in $\mathbb{P}^{1}$ containing a given point form a hyperplane and, as a divisor of degree $n$ cannot contain $n+1$ distinct points, no $n+1$ hyperplanes in the arrangement do meet.

In this case, we have more: for any natural number $n$ (not necessarily odd), we will show that any (ordered) $m$ hyperplane arrangement in $\mathbb{P}^{n}$ is projectively equivalent to a(n) (ordered) one arising from the above way. In fact, we will prove a stronger statement. Let $\mathfrak{M}_{1, n+3}$ be the moduli space of ordered $n+3$ distinct points in $\mathbb{P}^{1}$ and similarly $\mathfrak{M}_{n, n+3}$ ordered $n+3$ hyperplane arrangements in $\mathbb{P}^{n}$ in general position.

Lemma 3.5 The map $\gamma:\left(\mathbb{P}^{1}\right)^{n} \rightarrow \mathbb{P}^{n}$ induces an isomorphism

$$
\Gamma: \mathfrak{M}_{1, n+3} \simeq \mathfrak{M}_{n, n+3}, \quad\left[\left(p_{1}, \ldots, p_{m}\right)\right] \mapsto\left[\left(H_{1}, \ldots, H_{m}\right)\right]
$$


Proof We adopt an elementary but direct proof. The two moduli spaces admit affine descriptions. Any ordered $m=n+3$ distinct points in $\mathbb{P}^{1}$ is transformed into a unique tuple $\left(0, t_{1}, \ldots, t_{n-1}, \infty, 1, t_{n}\right)$ with $t_{i} \in \mathbb{C}$ and $\mathfrak{M}_{1, n+3}$ is therefore identified with the complement of the following hyperplanes in $\mathbb{C}^{n}=\operatorname{Spec} \mathbb{C}\left[t_{1}, \ldots, t_{n}\right]$ defined by

$$
\left\{t_{i}, t_{i}-1, t_{i}-t_{j}, 1 \leq i, j \leq n\right\} .
$$

Similarly, the following matrix represents a unique point in $\mathfrak{M}_{n, n+3}$ :

$$
\left(\begin{array}{ccccc}
1 & \ldots & 0 & 1 & 1 \\
0 & \ddots & 0 & 1 & s_{1} \\
\vdots & \ddots & \vdots & \vdots & \vdots \\
0 & \ldots & 1 & 1 & s_{n}
\end{array}\right)_{n+1 \times n+3},
$$

where $\left(s_{1}, \ldots, s_{n}\right)$ is a point in $\mathbb{C}^{n}=\operatorname{Spec} \mathbb{C}\left[s_{1}, \ldots, s_{n}\right]$ away from the union of hyperplanes

$$
\left\{s_{i}, s_{i}-1, s_{i}-s_{j}, 1 \leq i, j \leq n\right\} .
$$

The following claim implies the lemma:

Claim 3.6 Under the above affine coordinates, one has

$$
\Gamma\left(t_{1}, \ldots, t_{n-1}, t_{n}\right)=\left(\frac{t_{n}\left(t_{1}-1\right)}{t_{1}-t_{n}}, \ldots, \frac{t_{n}\left(t_{n-1}-1\right)}{t_{n-1}-t_{n}}, t_{n}\right) .
$$

Proof The following Vandermonde-type matrix of size $(n+1) \times(n+3)$ gives the defining equations of the hyperplane arrangement corresponding to

$$
\left(0, t_{1}, \ldots, t_{n-1}, \infty, 1, t_{n}\right)
$$

under $\gamma$ :

$$
A=\left(\begin{array}{ccccccc}
1 & 1 & \ldots & 1 & 0 & 1 & 1 \\
0 & t_{1} & \ldots & t_{n-1} & 0 & 1 & t_{n} \\
\vdots & \vdots & \vdots & \vdots & \vdots & \vdots & \vdots \\
0 & t_{1}^{n} & \ldots & t_{n-1}^{n} & 1 & 1 & t_{n}^{n}
\end{array}\right)
$$

The first $n+1$ columns make the square matrix

$$
B=\left(\begin{array}{ccccc}
1 & 1 & \ldots & 1 & 0 \\
0 & t_{1} & \ldots & t_{n-1} & 0 \\
\vdots & \vdots & \vdots & \vdots & \vdots \\
0 & t_{1}^{n} & \ldots & t_{n-1}^{n} & 1
\end{array}\right) .
$$

By Cramer's rule, one uses the determinant of a Vandermonde matrix to determine the vector

$$
\left(\lambda_{1}, \ldots, \lambda_{n+1}\right)^{t}=B^{-1}(1, \ldots, 1)^{t},
$$


as well as the vector

$$
\left(\mu_{1}, \ldots, \mu_{n+1}\right)^{t}=B^{-1}\left(1, t_{n}, \ldots, t_{n}^{n}\right)^{t} .
$$

Put $D=\operatorname{diag}\left\{\lambda_{1}, \ldots, \lambda_{\mathrm{n}+1}\right\}$. Then the invertible $(n+1) \times(n+1)$ matrix $P=$ $\lambda_{1} \mu_{1}^{-1} D^{-1} B^{-1}$ transforms the hyperplane arrangement defined by $A$ to the one by columns of the matrix

$$
\left(\begin{array}{ccccc}
1 & \ldots & 0 & 1 & 1 \\
0 & \ddots & 0 & 1 & \frac{t_{n}\left(t_{1}-1\right)}{t_{1}-t_{n}} \\
\vdots & \ldots & \vdots & \vdots & \vdots \\
0 & \ldots & 0 & 1 & \frac{t_{n}\left(t_{n-1}-1\right)}{t_{n-1}-t_{n}} \\
0 & \ldots & 1 & 1 & t_{n}
\end{array}\right)
$$

Let $p: C \rightarrow \mathbb{P}^{1}$ as above. The $n$-fold product

$$
h: C^{n} \stackrel{p^{n}}{\longrightarrow}\left(\mathbb{P}^{1}\right)^{n} \stackrel{\gamma}{\longrightarrow} \mathbb{P}^{n}
$$

is a Galois cover with Galois group $N \rtimes S_{n}$, where $N=\left\langle\iota_{1}, \ldots, \iota_{n}\right\rangle$ is the group generated by the cyclic automorphisms on factors. The group $N$ has a natural normal subgroup $N^{\prime}$ given by the kernel of the trace map

$$
N \simeq(\mathbb{Z} / r)^{\times n} \stackrel{\sum}{\longrightarrow} \mathbb{Z} / r
$$

It has a set of generators $\left\{\delta_{i}\right\}_{1 \leq i \leq r-1}$ with

$$
\delta_{i}=\left\langle i d, \ldots, \iota, \iota^{-1}, \ldots, i d\right\rangle,
$$

where $\iota$ appears at the $i$ th component and $\iota^{-1}$ the $i+1$ th component and the identity elsewhere. Consider the quotient $C^{n} / G$ with $G=N^{\prime} \rtimes S_{n}$. Similar to Lemma 2.8 loc. cit., one checks that the natural map $C^{n} / G \rightarrow \mathbb{P}^{n}$ induced by $h$ is a Galois cover with Galois group $\mathbb{Z} / r$ and its branch locus is exactly $H_{1}+\cdots+H_{m}$. As the Picard group of a projective space has no torsion, one concludes that $C^{n} / G$ is isomorphic to the $r$-fold cyclic cover $X_{C}$ of $\mathbb{P}^{n}$ branched along $\sum_{i} H_{i}$.

\subsection{The Abel-Jacobi Map and the Hodge Structure of the Cyclic Cover}

Recall that (see e.g. [4, Chap. 2]) the Abel-Jacobi map $\phi: C \rightarrow \operatorname{Jac}(C)=\mathbb{C}^{g} / \Lambda$ is defined by the period integral

$$
q \mapsto\left(\int_{q_{0}}^{q} \omega_{1}, \ldots, \int_{q_{0}}^{q} \omega_{g}\right),
$$

where $q_{0} \in C$ is a chosen base point and $\left\{\omega_{i}\right\}_{1 \leq i \leq g}$ is a basis of holomorphic one forms on $C$. Here $\Lambda$ denotes for the period lattice of $C$. Let $\left(z_{1}, \ldots, z_{g}\right)$ be 
the standard coordinates of $\mathbb{C}^{g}$. Then one sees that $\phi^{*}$ induces an isomorphism $H^{1}(\operatorname{Jac}(C), \mathbb{Q}) \simeq H^{1}(C, \mathbb{Q})$ such that $\phi^{*}\left(d z_{i}\right)=\omega_{i}, 1 \leq i \leq g$. The Abel-Jacobi map induces the natural morphism

$$
\phi_{n}: C^{n} \rightarrow \operatorname{Jac}(C), \quad\left(q_{1}, \ldots, q_{n}\right) \mapsto \sum_{i=1}^{n} \phi\left(q_{i}\right)
$$

We are in the situation to study the following diagram of morphisms:

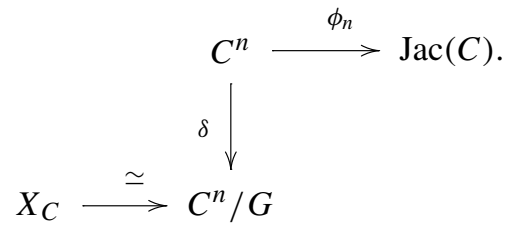

Here the map $\delta: C^{n} \rightarrow C^{n} / G$ is the natural projection. Note that after Lemma 3.5 any $r$-fold cyclic cover $X$ in Sect. 2.1 is isomorphic to an $X_{C}$. The consequence of the morphisms on the level of $\mathbb{Q}$-PHS is summarized in the following.

Proposition 3.7 The morphisms in the above diagram induces an isomorphism of Q-PHS:

$$
H^{n}\left(X_{C}, \mathbb{Q}\right) \simeq W_{1, \mathbb{Q}},
$$

where $W_{1, \mathbb{Q}}$ is the PHS in Lemma 3.3 .

Proof By labeling the curve factors of $C^{n}$ by $C_{i}, 1 \leq i \leq n$, we obtain from the Künneth decomposition a decomposition of $\mathbb{Q}$-PHS:

$$
\begin{aligned}
H^{n}\left(C^{n}, \mathbb{Q}\right) & =\bigotimes_{i=1}^{n} H^{1}\left(C_{i}, \mathbb{Q}\right) \\
& \oplus \bigotimes_{1 \leq i \neq j \leq n}\left[H^{0}\left(C_{i}, \mathbb{Q}\right) \otimes H^{2}\left(C_{j}, \mathbb{Q}\right) \otimes \bigotimes_{1 \leq k \leq n, k \neq i, j} H^{1}\left(C_{k}, \mathbb{Q}\right)\right] .
\end{aligned}
$$

Denote $\kappa: H^{n}\left(C^{n}, \mathbb{Q}\right) \rightarrow \bigotimes_{i=1}^{n} H^{1}\left(C_{i}, \mathbb{Q}\right)$ for the projection onto the first factor. We claim that the composite

$$
W_{1, \mathbb{Q}} \subset \bigwedge^{n} H^{1}(C, \mathbb{Q}) \stackrel{\phi^{*}}{\simeq} H^{n}(\operatorname{Jac}(C), \mathbb{Q}) \stackrel{\phi_{n}^{*}}{\longrightarrow} H^{n}\left(C^{n}, \mathbb{Q}\right) \stackrel{\kappa}{\longrightarrow} \bigotimes_{i=1}^{n} H^{1}\left(C_{i}, \mathbb{Q}\right)
$$

is injective and the image is $G$-invariant. Assuming the claim, the result follows. As

$$
H^{n}\left(X_{C}, \mathbb{Q}\right) \simeq H^{n}\left(C^{n} / G, \mathbb{Q}\right)=H^{n}\left(C^{n}, \mathbb{Q}\right)^{G}
$$


and $W_{1, \mathbb{Q}}$ have the same dimensions by Lemmas 3.1 and 3.2 (both are equal to $\left.2(r-1)^{2}\right)$, the injective map $W_{1, \mathbb{Q}} \hookrightarrow H^{n}\left(C^{n}, \mathbb{Q}\right)^{G}$ of $\mathbb{Q}$-PHSs is indeed an isomorphism. Notice that by Lemma 3.2 we can take the basis $\left\{\omega_{i}\right\}_{1 \leq i \leq g}$ in the above description of $\phi$ as eigenvectors with respect to the action of $\iota$ on $V_{\mathbb{C}}$. This implies that the corresponding $d z_{i}$ to $\omega_{i}$ is an eigenvector with the same eigenvalue with respect to the induced natural action of $\iota$ on $\operatorname{Jac}(C)$. This will make the verification of the invariance of the resulting class under $G$-action straightforward. Let $\pi_{i}: C^{n} \rightarrow C, 1 \leq i \leq n$ be the $i$ th projection. Then the induced map $\phi_{n}^{*}$ on the level of differential one forms is given by

$$
\phi_{n}^{*}\left(d z_{i}\right)=\sum_{l=1}^{n} \pi_{l}^{*} \omega_{i}, \quad \phi^{*}\left(d \bar{z}_{i}\right)=\sum_{l=1}^{n} \pi_{l}^{*} \bar{\omega}_{i}, \quad 1 \leq i \leq g .
$$

Therefore the map $\phi_{n}^{*}: H^{n}(\operatorname{Jac}(C), \mathbb{C}) \rightarrow H^{n}\left(C^{n}, \mathbb{C}\right)$ on the degree $n$ cohomology groups is given by sending $\left[d z_{i_{1}} \wedge \cdots \wedge d z_{i_{p}} \wedge d \bar{z}_{j_{1}} \wedge \cdots \wedge d \bar{z}_{j_{n-p}}\right]$ to

$$
\left[\sum_{l} \pi_{l}^{*} \omega_{i_{1}} \wedge \cdots \wedge \sum_{l} \pi_{l}^{*} \omega_{i_{p}} \wedge \sum_{l} \pi_{l}^{*} \bar{\omega}_{j_{1}} \wedge \cdots \wedge \sum_{l} \pi_{l}^{*} \bar{\omega}_{j_{n-p}}\right] .
$$

The bracket means the cohomology class of the differential form. Now for each direct factor $\bigwedge^{n} V_{k} \subset W_{1, \mathbb{Q}} \otimes \mathbb{Q}\left(\zeta_{r}\right)$, we claim that the image of its element under $\kappa \circ \phi_{n}^{*}$ is invariant under $G$-action. Note that the map $\phi_{n}$ factors as

$$
C^{n} \stackrel{/ S_{n}}{\longrightarrow} S^{n} C \rightarrow \operatorname{Jac}(C)
$$

Thus it suffices to show the invariant property under the subgroup $N^{\prime}$ of $G$. It is also equivalent to show this property for elements in $\bigwedge^{n}\left(V_{k} \otimes \mathbb{C}\right)$, which for dimension reason, is just equal to

$$
\bigwedge^{2 k-2} V_{k}^{1,0} \otimes \bigwedge^{n-2 k+2} V_{k}^{0,1} \oplus \bigwedge_{k}^{2 k-1} V_{k}^{1,0} \otimes \bigwedge^{n-2 k+1} V_{k}^{0,1}
$$

Let $\left\{d z_{i_{1}}, \ldots, d z_{i_{2 k-1}}\right\}$ (resp. $\left\{d \bar{z}_{j_{1}}, \ldots, d \bar{z}_{j_{n-2 k+2}}\right\}$ ) be the basis of $V_{k}^{1,0}$ (resp. $V_{k}^{0,1}$ ). Consider the image under $\phi_{n}^{*}$ of a typical element (omitting $d z_{i_{2 k-1}}$ in the wedge product)

$$
[\alpha]:=\left[d z_{i_{1}} \wedge \cdots \wedge d z_{i_{2 k-2}} \wedge d \bar{z}_{j_{1}} \wedge \cdots d \bar{z}_{j_{n-2 k+2}}\right] \in \bigwedge^{2 k-2} V_{k}^{1,0} \otimes \bigwedge^{n-2 k+2} V_{k}^{0,1} .
$$

For clarity we set

$$
\alpha_{1}=\omega_{i_{1}}, \quad \ldots, \quad \alpha_{2 k-2}=\omega_{i_{2 k-2}}, \quad \alpha_{2 k-1}=\bar{\omega}_{j_{1}}, \quad \ldots, \quad \alpha_{n}=\bar{\omega}_{j_{n-2 k+2}} .
$$

Then it follows that

$$
\kappa \circ \phi_{n}^{*}([\alpha])=\sum_{\nu \in S_{n}}(-1)^{\operatorname{Sign}(v)}\left[\pi_{1}^{*} \alpha_{\nu(1)} \wedge \cdots \wedge \pi_{n}^{*} \alpha_{\nu(n)}\right] .
$$


(For $n=3 \bmod 4$, we have $\kappa \circ \phi_{n}^{*}([\alpha])=\phi_{n}^{*}([\alpha])$.) Now one sees its invariance under $N^{\prime}$-action immediately. One tests simply the action of any generator $\delta_{i}$ of $N^{\prime}$ and it acts on $\pi_{1}^{*} \alpha_{\nu(1)} \wedge \cdots \wedge \pi_{n}^{*} \alpha_{\nu(n)}$ by multiplying $\zeta_{r}^{k} \zeta_{r}^{-k}=1$. This completes the proof.

\section{Maximal Families of CY Manifolds with Length 1 Yukawa Coupling}

Our aim in this section is to exhibit families of CY manifolds with claimed properties, and make some complements to these families at the end.

Recall that in the proof of Lemma 3.5 we have explained that $\mathfrak{M}_{n, n+3}$ is identified with an open subset of $\mathbb{C}^{n}$. Call it $U_{n}$ with coordinates $s=\left(s_{1}, \ldots, s_{n}\right)$. Let $\left[x_{0}: \cdots\right.$ : $x_{n}$ ] be the homogeneous coordinates of $\mathbb{P}^{n}$. Put

$$
t_{1}=x_{0}, \quad \ldots, \quad t_{m-2}=x_{n}, \quad t_{m-1}=\sum_{i=0}^{n} x_{i}, \quad t_{m}=x_{0}+\sum_{i=1}^{n} s_{i} x_{i} .
$$

They give $m$ sections of $\mathcal{O}(1)$ whose zero divisors in $\mathbb{P}^{n}$ meet transversally. Let $p_{i}, i=1,2$ be the projection of $\operatorname{Tot}(L) \times U_{n}$ to the $i$ th factor. Define

$$
\mathcal{X}_{n} \subset \operatorname{Tot}(L) \times U_{n}
$$

to be the zero locus of the section

$$
p_{1}^{*} s^{r}-\bigotimes_{i=1}^{m}\left(p \circ p_{1}\right)^{*} t_{i} \in \Gamma\left(\operatorname{Tot}(L) \times U_{n},\left(p \circ p_{1}\right)^{*} \mathcal{O}(m)\right),
$$

and $f_{n}: \mathcal{X}_{n} \rightarrow U_{n}$ the composite $\mathcal{X}_{n} \subset \operatorname{Tot}(L) \times U_{n} \stackrel{p_{2}}{\rightarrow} U_{n}$. The is the family of $r$-fold cyclic covers of $\mathbb{P}^{n}$ branched along a universal family of $n+3$ hyperplane arrangements of $\mathbb{P}^{n}$ in general position. We can do the simultaneous crepant resolution of the family $f_{n}$. The divisors of $\operatorname{Tot}(L) \times U_{n}$ given by

$$
\tilde{E}_{0}:=\left(p_{1}^{*} s=0\right), \quad \tilde{E}_{1}:=\left(\left(p \circ p_{1}\right)^{*} t_{1}=0\right), \quad \ldots, \quad \tilde{E}_{m}:=\left(\left(p \circ p_{1}\right)^{*} t_{m}=0\right)
$$

meet transversally and the hypersurface $\mathcal{X}$ is binomial with respect to

$$
\left(\mathfrak{E}=\left\{\tilde{E}_{0}\right\}, \mathfrak{F}=\left\{\tilde{E}_{1}, \ldots, \tilde{E}_{m}\right\}\right) .
$$

By Theorem 2.5, we can apply the crepant resolution algorithm to $\mathcal{X}$ and get a simultaneous crepant resolution of $f_{n}$ :

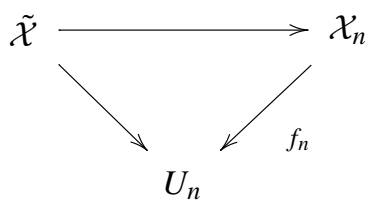


This is not unique and a different choice leads to a fiberwise birationally equivalent family of CY manifolds. We choose one and call $\tilde{f}_{n}: \tilde{\mathcal{X}}_{n} \rightarrow U_{n}=\mathfrak{M}_{n, n+3}$ the family of CY manifolds by our construction.

We can also consider $\mathfrak{M}_{1, n+3}$ and obtain a family $g_{n}: \mathcal{C} \rightarrow \mathfrak{M}_{1, n+3}$ whose fibers are $r$-fold cyclic covers of $\mathbb{P}^{1}$ branched along $n+3$ distinct points. The $n$th self product $\left(g_{n}\right)^{n}: \mathcal{C}^{n} \rightarrow \mathfrak{M}_{1, n+3}$ of $g_{n}$ admits a natural action of $G$ which acts fiberwisely as what we have described at the end of Sect. 3.1. For

$$
h_{n}: \mathcal{C}^{n} / G \stackrel{\overline{\left(g_{n}\right)^{n}}}{\longrightarrow} \mathfrak{M}_{1, n+3} \stackrel{\Gamma}{\longrightarrow} \mathfrak{M}_{n, n+3},
$$

there is an isomorphism

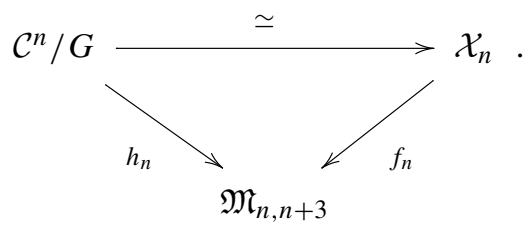

Some notations before the main computational result. Write $\mathbb{V}_{\mathbb{Q}}=R^{1} g_{n *} \mathbb{Q}$. By Lemma 3.2, the fiberwise cyclic automorphism induces a decomposition of $\mathbb{Q}\left(\zeta_{r}\right)$ PVHS:

$$
\mathbb{V}_{\mathbb{Q}} \otimes \mathbb{Q}\left(\zeta_{r}\right)=\bigoplus_{i=1}^{r-1} \mathbb{V}_{i}
$$

By Lemma 3.3, there is a decomposition of $\mathbb{Q}$-PVHSs:

$$
\bigwedge^{n} \mathbb{V}_{\mathbb{Q}}=\mathbb{W}_{1, \mathbb{Q}} \oplus \mathbb{W}_{2, \mathbb{Q}}
$$

such that $\mathbb{W}_{1, \mathbb{Q}} \otimes \mathbb{Q}\left(\zeta_{r}\right)=\bigoplus_{i=1}^{r-1} \bigwedge^{n} \mathbb{V}_{i}$. Write $\mathbb{H}_{n}=R^{n} \tilde{f}_{n *} \mathbb{Q}$.

Theorem 4.1 Let $\tilde{f}_{n}: \tilde{\mathcal{X}}_{n} \rightarrow \mathfrak{M}_{n, n+3}$ be the family of $n$-dimensional CY manifolds. Then one has an isomorphism of $\mathbb{Q}$-PVHSs:

$$
\mathbb{H}_{n} \simeq \mathbb{W}_{1, \mathbb{Q}}
$$

Proof It follows from Propositions 2.8 and 3.7.

Remark 4.2 Note that, for and only for $n=3,5,9$, the $\mathbb{Q}\left(\zeta_{r}\right)$-PVHS

$$
\bigwedge^{n} \mathbb{V}_{1} \oplus \bigwedge^{n} \mathbb{V}_{r-1}
$$

underlies a sub $\mathbb{Q}$-PVHS $\mathbb{W}_{\text {unif, } \mathbb{Q}}$ of $\mathbb{W}_{1, \mathbb{Q}}$. Write $\mathbb{H}_{\text {unif, } \mathbb{Q}}$ to be the sub $\mathbb{Q}$-PVHS of $\mathbb{H}_{n}$ corresponding to $\mathbb{W}_{\text {unif, } \mathbb{Q}}$ under the above isomorphism. 
Corollary 4.3 The following statements are true:

(i) The family $\tilde{f}_{n}$ is maximal.

(ii) $\varsigma\left(\tilde{f}_{n}\right)=1$. Consequently, Shafarevich's conjecture holds true for $\tilde{f}_{n}$.

(iii) A suitable partial compactification of the family $\tilde{f}_{3}$ is a Shimura family of $U(1,3)$-type.

(iv) For $n=5,9$, the sub $\mathbb{Q}$-PVHS $\mathbb{H}_{\text {unif, } \mathbb{Q}} \subset \mathbb{H}_{n}$ gives a uniformization to a Zariski open subset of an arithmetic ball quotient.

Proof Let $(F, \eta)$ be the corresponding Higgs bundle to $\mathbb{V}_{\mathbb{Q}},(E, \theta)$ to $\mathbb{H}_{n}$. The above theorem on PVHS implies the corresponding result on Higgs bundles, that is,

$$
(F, \eta)=\bigoplus_{i=1}^{r-1}\left(F_{i}, \eta_{i}\right), \quad(E, \theta) \simeq \bigoplus_{i=1}^{r-1} \bigwedge^{n}\left(F_{i}, \theta_{i}\right)
$$

Lemma 3.2 implies that

$$
\operatorname{rank}\left(F_{i}^{1,0}\right)=2 i-1, \quad \operatorname{rank}\left(F_{i}^{0,1}\right)=n+2-2 i .
$$

Adding the information on the Hodge components, one has a more explicit description of the Higgs bundle $(E, \theta)$, which is equal to

$$
\begin{aligned}
& \left(E^{n, 0} \oplus E^{n-1,1}, \theta^{n, 0}\right) \\
& \quad \oplus\left(E^{n-2,2} \oplus E^{n-3,3}, \theta^{n-2,2}\right) \oplus \cdots \oplus\left(E^{1, n-1} \oplus E^{0, n}, \theta^{1, n-1}\right),
\end{aligned}
$$

together with isomorphisms of Higgs bundles

$$
\begin{aligned}
\left(E^{n, 0} \oplus E^{n-1,1}, \theta^{n, 0}\right) & \simeq\left(\bigwedge^{n} F_{r-1}^{1,0} \oplus \bigwedge^{n-1} F_{r-1}^{1,0} \otimes F_{r-1}^{0,1}, \bigwedge^{n} \eta_{r-1}\right), \\
& \ldots \\
\left(E^{1, n-1} \oplus E^{0, n}, \theta^{1, n-1}\right) & \simeq\left(\bigwedge^{n-1} F_{1}^{1,0} \otimes F_{1}^{0,1} \oplus \bigwedge^{n} F_{1}^{0,1}, \bigwedge^{n} \eta_{1}\right) .
\end{aligned}
$$

Note that the family $g_{n}: \mathcal{C} \rightarrow \mathfrak{M}_{1, n+3}$ is in connection with the theory of DeligneMostow [1] (see also [6]). It follows from Proposition 3.9 loc. cit. that

$$
\eta_{r-1}: F_{r-1}^{1,0} \rightarrow F_{r-1}^{0,1} \otimes \Omega_{\mathfrak{M}_{1, n+3}}
$$

is an isomorphism. So is $\theta^{n, 0}$. This shows (i). It follows also that $\varsigma\left(\tilde{f}_{n}\right)=1$ as

$$
\theta^{n-1,1}: E^{n-1,1} \rightarrow E^{n-2,2} \otimes \Omega_{\mathfrak{M}_{n, n+3}}
$$

is simply zero. As $\tilde{f}_{n}$ is maximal, the length of the Yukawa coupling of the corresponding coarse moduli is also equal to 1 . By [5, Theorem 6], Shafarevich's conjecture holds for this coarse moduli. This shows (ii). The eigen-PVHS $\mathbb{V}_{1}$ is a special 
case of $\mathbb{C}$-PVHSs over $\mathfrak{M}_{1, n+3}$ studied by Deligne-Mostow loc. cit. In our case,

$$
\mu=\left(\mu_{1}=\frac{1}{r}, \ldots, \mu_{m}=\frac{1}{r}\right)
$$

in the notation of loc. cit. It is shown in Sect. 10 loc. cit. that when the condition INT (see Theorem $3.11 \mathrm{loc}$. cit. for its meaning) is satisfied, the monodromy representation of $\mathbb{V}_{1}$ has discrete image in $\operatorname{Aut}\left(\mathbb{B}^{n}\right)$. The condition to guarantee the discreteness of the image has been relaxed by Mostow [8] to $\sum$ INT, which turns out to be also sufficient by Mostow [9]. In the appendix of [9], he gives also a complete list of $\mu$ satisfying $\sum$ INT for $n \geq 3$. Checking the list, one finds immediately that there are exactly three cases labeled as No. 1, No. 8 and No. 23 which have equal $\mu_{i}$ s, and they come exactly from $\mathbb{V}_{1}$ for $n=3,5,9$. Moreover, in these three cases, the monodromy groups are actually arithmetic. Note that

$$
\mathbb{H}_{\text {unif }, \mathbb{Q}} \otimes \mathbb{Q}\left(\zeta_{r}\right) \simeq\left(\mathbb{V}_{1} \oplus \mathbb{V}_{r-1}\right) \otimes \mathbb{Q}\left(\zeta_{r}\right)(2-r)
$$

and $\mathbb{V}_{1}, \mathbb{V}_{r-1}$ are dual to each other. The period map of $\mathbb{H}_{\text {unif, } \mathbb{Q}}$ gives an open embedding of $\mathfrak{M}_{n, n+3}$ into an arithmetic ball quotient. For the case $n=3$, it is even more special. In this case, one has

$$
\mathbb{H}_{3}=\mathbb{H}_{\text {unif, } \mathbb{Q}} \simeq \mathbb{V}_{\mathbb{Q}} \otimes \mathbb{Q}(-1)
$$

By Deligne-Mostow [1], the family $g_{3}: \mathcal{C} \rightarrow \mathfrak{M}_{1,6}$ can be partially compactified so that the image of its period map in the moduli space of principally polarized Abelian 4-folds with a suitable level structure is an arithmetic quotient of $\mathbb{B}^{3}$. See a recent work [7] by Moonen on the classification of Shimura subvarieties in the Jacobian locus of moduli spaces of principally polarized Abelian varieties arising from cyclic covers of $\mathbb{P}^{1}$. This example appears as No. (10) in Table 1 loc. cit. It is quite obvious that the partial compactification of $g_{3}$ yields the one of $f_{3}$ and also of $\tilde{f}_{3}$. The detail is omitted since what it will involve is not closely relevant to the paper. This completes the proof.

We conclude the paper with the following remark.

Remark 4.4 (i) For $n=3$, the Hodge numbers of $\tilde{X}$ read $h^{1,1}=51, h^{2,1}=3$. Rohde has constructed in his doctor thesis (see [11]) a maximal family of CY 3-folds with the same Hodge numbers which is also a Shimura family. Note that the parameter of his family comes also from $\mathfrak{M}_{1,6}$. Are these two families birationally equivalent?

(ii) One constructs more maximal families of CY manifolds from the moduli space of hyperplane arrangements in a projective space. Do our families exhaust all possibilities with length 1 Yukawa coupling?

(iii) We have shown that $\mathbb{H}_{\text {unif, } \mathbb{Q}} \subset \mathbb{H}_{n}$ exists only for $n=3,5$, 9, which are the unique three cases appeared in Mostow's list with equal $\mu_{i} \mathrm{~s}$. Is there some deeper reason than a mere coincidence? 
Acknowledgements This work is supported by the SFB/TR 45 'Periods, Moduli Spaces and Arithmetic of Algebraic Varieties' of the DFG, and partially supported by the University of Science and Technology of China.

We would like to thank Guitang Lan for helpful discussions, particularly in Lemma 3.5. Our special thanks go to Igor Dolgachev who has drawn our attention to the $n=3$ case of the paper.

\section{References}

1. Deligne, P., Mostow, G.D.: Monodromy of hypergeometric functions and non-lattice integral monodromy. Publ. Math. IHÉS 63, 5-89 (1986)

2. Dolgachev, I., Kondō, S.: Moduli of K3 surfaces and complex ball quotients. In: Arithmetic and Geometry Around Hypergeometric Functions. Lecture Notes of a CIMPA Summer School, held at Galatasaray University, Istanbul (2005)

3. Gerkmann, R., Sheng, M., van Straten, D., Zuo, K.: On the monodromy of the moduli space of CalabiYau threefolds coming from eight planes in $\mathbb{P}^{3}$. Math. Ann. (2012). doi:10.1007/s00208-012-0779-z

4. Griffiths, P., Harris, J.: Principles of Algebraic Geometry. Pure and Applied Mathematics. WileyInterscience, New York (1978)

5. Liu, K.-F., Todorov, A., Yau, S.-T., Zuo, K.: Finiteness of subfamilies of Calabi-Yau $n$-folds over curves with maximal length of Yukawa coupling. Pure Appl. Math. Q. 7(4), 1585-1598 (2011)

6. Looijenga, E.: Uniformization by Lauricella functions an overview of the theory of Deligne-Mostow, arithmetic and geometry around hypergeometric functions. Prog. Math. 260, 207-244 (2007)

7. Moonen, B.: Special subvarieties arising from families of cyclic covers of the projective line (2010). arXiv:1006.3400v2

8. Mostow, G.D.: Generalized Picard lattices arising from half-integral conditions. Publ. Math. IHÉS 63, 91-106 (1986)

9. Mostow, G.D.: On discontinuous action of monodromy groups on the complex $n$-ball. J. Am. Math. Soc. 1(3), 555-586 (1988)

10. Peters, C., Steenbrink, J.: Mixed Hodge Structures. Ergebnisse der Mathematik und ihrer Grenzgebiete. 3., vol. 52. Springer, Berlin (2008)

11. Rohde, J.: Cyclic coverings, Calabi-Yau manifolds and complex multiplication. Lecture Notes in Mathematics. Springer, Berlin (1975). 2009

12. Terasoma, T.: Infinitesimal variation of Hodge structures and the weak global Torelli theorem for complete intersections. Ann. Math. 132(2), 213-225 (1990)

13. Viehweg, E., Zuo, K.: Complex multiplication, Griffiths-Yukawa couplings, and rigidity for families of hypersurfaces. J. Algebr. Geom. 14(3), 481-528 (2005)

14. Zhang, Y.: Rigidity for families of polarized Calabi-Yau varieties. J. Differ. Geom. 68(2), 185-222 (2004) 